1999-01-01

\title{
Spin-up in a tank induced by a rotating bluff body
}

R. Daniel Maynes

J. Klewicki

P. McMurty

Follow this and additional works at: https://scholarsarchive.byu.edu/facpub

Part of the Mechanical Engineering Commons

Original Publication Citation

Journal of Fluid Mechanics 388(1999): 49-68.

\section{BYU ScholarsArchive Citation}

Maynes, R. Daniel; Klewicki, J.; and McMurty, P., "Spin-up in a tank induced by a rotating bluff body" (1999). Faculty Publications. 628.

https://scholarsarchive.byu.edu/facpub/628

This Peer-Reviewed Article is brought to you for free and open access by BYU ScholarsArchive. It has been accepted for inclusion in Faculty Publications by an authorized administrator of BYU ScholarsArchive. For more information, please contact ellen_amatangelo@byu.edu. 


\title{
Spin-up in a tank induced by a rotating bluff body
}

\author{
By D. MAYNES ${ }^{1} \dagger$, J. KLEWICKI ${ }^{2}$ \\ AND P. MCMURTR Y \\ ${ }^{1}$ Department of Mechanical Engineering, Brigham Young University, Provo, UT 84602, USA \\ ${ }^{2}$ Department of Mechanical Engineering, University of Utah, SLC, UT 84112, USA
}

(Received 11 November 1997 and in revised form 21 December 1998)

Spin-up of a turbulent flow in a cylindrical tank caused by a rotating bluff body has been investigated using flow visualization, fluid velocity measurements, and hydrodynamic torque measurements. During the spin-up process three distinct temporal regimes exist. These regimes are: (i) a build-up regime where the torque and the tangential velocity fluctuations in the close proximity of the body remain constant; (ii) a decay regime where these quantities decay with power-law relations; and (iii) a mean flow steady state where these values remain relatively constant. Experiments were conducted in two tanks differing in volume by a factor of 80 and with a large range of bluff body sizes. A non-dimensional time scale, $\tau$, based upon turbulent diffusion is determined and the tangential velocity fluctuations and torque coefficient start to decay at a fixed value of $\tau$. Likewise, steady state is attained at a larger fixed value of $\tau$. This time scaling is physically based upon the time required for momentum to be transferred over the entire tank volume due to turbulent diffusion, and is general for any body size, tank size, rotation rate, and acceleration rate.

\section{Introduction}

The spin-up of a laminar fluid caused by the rotation of a cylindrical tank has previously been addressed (Wedemeyer 1964; Greenspan 1968). It has been shown that for spin-up the flow consists of three distinct phases: the formation of an Ekman boundary layer, secondary flow, and the viscous decay of small residual oscillations. Secondary flow accounts for the advective transport of angular momentum from the walls to the interior and consequently the flow evolves to solid-body rotation several orders of magnitude faster than for flow in an infinite length tank. Greenspan (1968) showed that the time for spin-up to steady state for this configuration is proportional to $\left(H^{2} /(\omega v)\right)^{1 / 2}$ where $H$ is the tank and fluid height, $\omega$ is the rotation rate, and $v$ is the kinematic viscosity. This expression is limited, however, to the case of a rotating tank and laminar flow. The flow around a rotating bluff body (non-circular) in a tank is significantly different from flow inside a rotating cylinder. Three major distinctions are: the rotating body drives the fluid motion instead of the tank walls, large separated regions exist along the faces of a bluff body, and the flow field is turbulent except at very low Reynolds number $(\sim 100)$. Flow fields associated with rotating bluff bodies in a tank also differ fundamentally from flow fields associated with uniform flow past bluff bodies. Flow fields of the latter type result in a wake that

$\dagger$ Author to whom correspondence should be addressed: e-mail maynesrd@et.byu.edu. 
convects downstream from the point of flow separation on the body. At moderate to high $R e$, uniform flow past bluff bodies results in periodic vortex shedding on the leeside of the body. Conversely, a rotating bluff body in a tank rotates in its own wake and the flow field is dominated by large-scale (order body size) coherent vortical motions. For all rotating bluff bodies the flow field is also highly three-dimensional. Thus during start-up the flow field associated with a rotating bluff body is unsteady, separated, three-dimensional, and turbulent.

One common flow field of this type is the mixing tank common in most processing industries and numerous researchers have addressed the flow in stirred tanks. Of particular interest has been the turbulence in the bulk flow and in the impeller stream (Wu \& Patterson 1989; Zhou \& Kresta 1996; Costes \& Couderc 1988), the scale up of geometrically similar tanks (Rice \& Baud 1990; Bourne \& Dell'Ava 1987) and the effects of impellers of different geometries on the flow field characteristics (Rutherford et al. 1996; Gunkel \& Weber 1975; Roberts et al. 1995). Most of the literature addressing stirred tanks, however, focuses on geometric ratios of the impeller radius to tank radius of $0.33-0.50$ and impeller blade height to tank height of $0.05-$ 0.07. A review of the literature also indicates that the transient evolution from a quiescent fluid to steady state (mean velocity field) has been largely unexplored. This is because in practice stirred mixing tanks operate in a steady-state (unidirectional rotation) environment.

Applications exist, however, where the behaviour of the flow field during the transient process is more important than the steady-state flow field. One such application is a modified accelerated crucible technique (Zaitseva 1997) employed for the growth of large KDP crystals from solution. Periodic mixing processes also fall in this category. Thus, the present work focuses on the evolution of the turbulent flow field from start-up to steady state over a range of bluff body sizes where the ratio of body height to tank height is much larger than those typically explored for stirred mixing tanks. Specifically, this paper reports on a general description of the evolving turbulent flow field around rotating bluff bodies from start-up to steady state; and on the development of a time scale which describes spin-up for rotating bluff bodies that is general for all body sizes and tank sizes investigated. The investigation was experimental in nature and employed flow visualization, time-resolved measurements of the tangential velocity, and time-resolved measurements of the hydrodynamic torque.

\section{Experimental setup and techniques}

\subsection{Experimental configuration}

Experiments were conducted in two flow facilities. The first facility, hereafter referred to as the small tank, is $0.254 \mathrm{~m}$ in height and $0.219 \mathrm{~m}$ in diameter. The second flow facility, hereafter referred to as the big tank, is $1.524 \mathrm{~m}$ in height and has an inside diameter of $0.914 \mathrm{~m}$. The liquid used in both facilities was distilled water at about $20^{\circ} \mathrm{C}$. For each facility, an in-line motor was mounted directly above the tank and connected to a shaft attached to the body of interest. The motors were controlled by a PC terminal via indexer drives. A schematic of a bluff body in a tank with the tank and body dimensions labelled is shown in figure 1. This figure also depicts the optical and camera setup for the velocity measurement technique employed (molecular tagging velocimetry). The characteristic length, $L$, of the bodies is the distance from the centre to a corner in a horizontal plane $(r, \theta)$ or $L=\sqrt{2}(F / 2)$ where $F$ is the face length. The characteristic length of the tank is the radius, $R$, and the tank height 

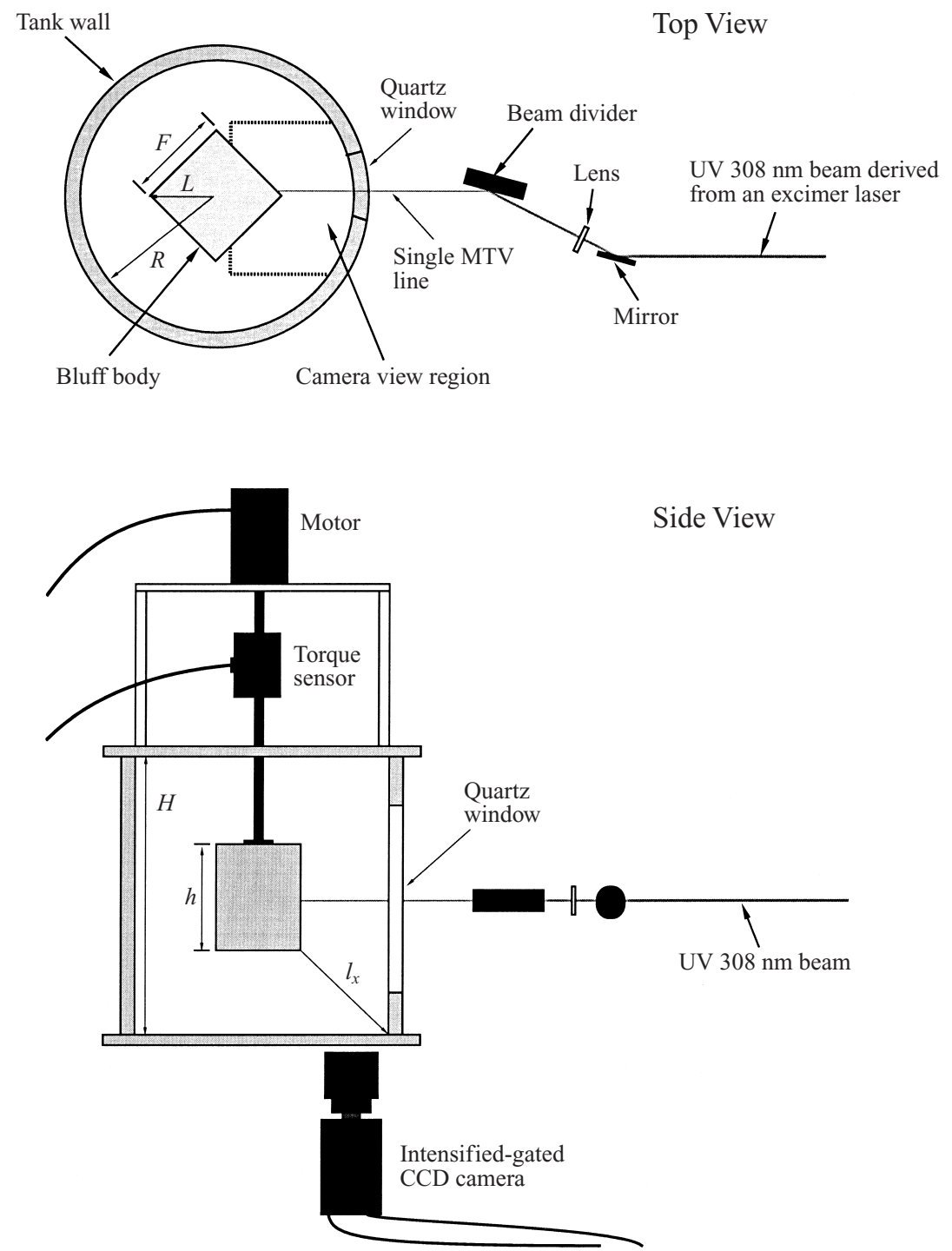

FIgURE 1. Schematic of a flow facility showing a typical bluff body, motor, in-line torque sensor, and MTV optical and camera setup.

and body height are denoted as $H$ and $h$ respectively. Two bluff bodies were used in the small tank with $L^{*}=L / R=0.52$ and 0.65 and $h^{*}=h / R=0.85$ for both bodies. Seven bluff bodies were used in the large tank. The sizes of these bodies were $L^{*}=0.13,0.15,0.23,0.23,0.31,0.39$, and 0.7 and $h^{*}=2.1,1.05,1.05,0.38,0.48,1.05$, and 0.72 respectively. The bodies were vertically centred in the tank unless otherwise noted. Rotation rates, $\omega$, ranging from 0.2 r.p.s. to 2.0 r.p.s. and accelerations, $\alpha$, ranging from 0.01 r.p.s. ${ }^{2}$ to 10.0 r.p.s. ${ }^{2}$ were investigated. For most cases, both tanks were completely full so that a free surface does not exist. Exceptions to this will be noted.

\subsection{Flow visualization}

For the flow visualization experiments the flow was seeded with 2-10 $\mu \mathrm{m}$ particles that phosphoresce when excited by UV light. These particles are produced by GTE 
and are Sylvania type 2363. A $308 \mathrm{~nm}$ wavelength sheet of light (derived from a Lumonics Ex-700 excimer laser) was used to excite the particles. The same laser was used in the velocity measurements. A gated intensified video camera mounted perpendicular to the plane of the laser sheet and synchronized so that it shutters immediately after the laser fires was used to acquire the visual data. The exposure time of each video image was large enough to generate particle streaks. Because these streaks are asymmetric (owing to the decaying phosphorescence) velocity direction and flow structure are apparent from single frames. Thus phosphorescent particles have a distinct advantage over particles that only reflect incident light.

Flow visualization experiments were conducted in both the large and small tanks over a wide range of $\alpha$ and $\omega$. Regardless of the body used or rotation parameters chosen, flow visualization experiments were conducted in the following manner. After the fluid had completely come to rest, a sheet of UV laser light illuminated the phosphorescent particles in a region of interest and images were recorded on a SVHS tape. Motion was initiated by the motor controller and images were recorded at $30 \mathrm{~Hz}$ thus giving a video of the flow field in a fixed spatial plane. Visualizations were conducted both in the $(r, \theta)$ - and $(r, z)$-planes. The $(r, \theta)$-plane images were obtained by positioning the camera below the tank and focusing through a window on an illuminated $(r, \theta)$-plane.

\subsection{Molecular tagging velocimetry (MTV)}

A relatively new technique was used for the measurement of fluid velocities. Following the suggestion of Gendrich \& Koochesfahani (1996) the method is most generally called molecular tagging velocimetry or MTV (formerly it was called laser-induced photochemical anemometry or LIPA). This technique has evolved from the first fluid mechanics applications of light sensitive chemicals in 1962 (Miller 1962) to a powerful velocity measurement tool with contributions and improvements made by a number of researchers (Falco \& Chu 1987). MTV conceptually and in practice is straightforward. The flow field of interest is first doped with a small concentration of photochemical. For this experimental programme the photoluminescent chemical (1-BrNp•Gb-CD•ROH ternary complex), developed by Ponce et al. (1993), was used. Using a Xybion ISG-250 camera, this chemical has an imageable phosphorescent 'life time' of about $12 \mathrm{~ms}$.

The optical configuration used to excite the dissolved photochemical involves dividing the UV laser beam into a series of approximately parallel lines. The hardware and procedures for using this version of MTV is given in detail by Hill \& Klewicki (1996). A brief synopsis is presented here. The laser fires, exciting the photochemical along the lines. The lines are then allowed to deform as the fluid flows. After a few milliseconds a synchronized CCD camera shutters, capturing the deformed lines on video. The distance that each point along a line deforms is measured by comparing the undeformed and deformed lines. The velocity component normal to a line can be calculated by $V \approx \Delta x / \Delta t$, where $\Delta x$ is the distance between deformed and undeformed lines and $\Delta t$ is the time between the laser pulse and the time the camera shutters. The velocity can be computed as above for each pixel along the set of laser lines. The result is a set of instantaneous velocity profiles for each frame recorded by the camera. A single MTV line was used for the tangential velocity profile measurements and a schematic of the experimental setup for the MTV measurements is shown in figure 1. Errors in measuring the velocities in both tanks are typically on the order of 3.5-6.0\% for instantaneous velocities (Hill \& Klewicki 1996). The spatial resolution for measurements in the large tank $\left(2.704 \times 10^{-4} \mathrm{~m} /\right.$ pixel $)$ was typically one third the 
spatial resolution $\left(9.54 \times 10^{-5} \mathrm{~m} / \mathrm{pixel}\right)$ in the small tank. The radial length of the MTV profiles was about $0.08 \mathrm{~m}$ in the large tank and $0.025 \mathrm{~m}$ in the small tank.

\subsection{Hydrodynamic torque measurements}

The hydrodynamic torque was measured using an in-line torque transducer and the experimental procedure, including bluff body inertia and frictional effects, have been previously discussed (Maynes, Klewicki \& McMurtry 1998).

\section{Discussion of torque measurements and flow visualization observations}

A common measurement in stirred mixing tanks is the power required to maintain stirring at a constant rate. The power is the product of the hydrodynamic torque, $T$, and the rotation rate, $\omega$. At steady state the power is balanced by the rate of energy dissipation within the tank. During spin-up from rest, however, the measured torque is indicative of the different fluid mechanics regimes which exist in the transient process. Transient torque measurements on rotating bluff bodies indicate that three distinct temporal regimes exist from start-up to steady state (Maynes et al. 1998). Steady state refers to the mean velocity field and mean hydrodynamic torque. That work presented measurements of the hydrodynamic torque on rotating bluff bodies over an $L^{*}$-range of $0.15-0.7$ and an $h / H$-range of 0.15-0.63. These measurements were conducted in the large tank described above. The three temporal regimes were referred to as the build-up, decay, and steady-state regimes. The build-up regime begins upon initiation of motion, and the torque is approximately constant for the duration of the build-up regime. At a certain number of revolutions, $t^{*}$, dependent upon the body size, the torque begins to decay with a power law relation $T \sim t^{*-n}$. The exponent, $n$, was found to be approximately 1 for $\operatorname{Re}=\left(\omega L^{2} / v\right) \geqslant 7 \times 10^{4}$, but decreases as $\operatorname{Re}$ decreases. These torque measurements show that for a fixed body size, the decay regime begins at a constant number of revolutions regardless of $\alpha$ or the final rotation rate, $\omega_{f}$, provided $\alpha$ is sufficiently high. Additionally, it was shown that steady state is attained at a fixed number of revolutions. Fluctuations in the torque were observed due to the nature of turbulent flow. These torque measurements further show that the decay regime begins at a fixed value of $t_{d}=t^{*} L^{2} h /\left(R^{2} H\right)$, which was shown to be general for all body sizes investigated with the tank size held constant. This value is about 0.5 . Steady state occurs at a value of $t_{d}$ of about 3.0. If the height of the tank is varied, the steady state that is attained varies in a proportional manner. Based on these torque results there are likely to exist analogous temporal regimes in the velocity field.

The flow visualization technique described above was also used to give a qualitative description of the flow field after start-up. These visualization studies were conducted at $(r, \theta)$ - and $(r, z)$-planes near the body (including the rotating body edge) and near the tank walls. Results from these visualizations in the $(r, \theta)$-plane indicate that upon initiation of rotation, a vortex rolls up at each body corner. This vortex convects outward and subsequently interacts with other eddies and the bulk fluid. For sufficiently high $\alpha$ the rolled-up vortex interacts with the bulk fluid. If $\alpha$ is not sufficient, the initial vortex interacts with the next rotating corner. In either case turbulent motions are evident throughout the tank. Transient flow field evolution occurs for continuing body rotation and coherent, separated, recirculation regions from adjacent to the body faces. These separated regions extend radially to $O(L)$. A shear layer is evident and remains coherent at the interface between the recirculation region and the bulk flow. As the body continues to rotate, the fluid outside the 
separated region, although starting an overall rotation, consists of a collection of randomly interacting eddies. The time over which this behaviour occurs corresponds to the build-up regime from the hydrodynamic torque measurements. Recall that the hydrodynamic torque remains constant in this regime. This behaviour continues for a time that is dependent upon body size until the fluid motion appears to develop a strong solid-body-rotation-like component. From the torque history, this corresponds to the decay regime. Physically, decay regime flow dynamics are associated with a decrease in the radial extent of the recirculation regions. During the decay regime the interacting eddies and the recirculation regions decrease in size and the torque decays with a power law of the form $T \sim t^{*-n}$ until the mean flow steady state is attained. At this time the torque levels off to a constant mean value. Also, the size of the recirculation regions are at a minimum and remain relatively constant in size.

Flow visualization in the $(r, z)$-plane indicates that for a short time after start-up the flow is not affected near the tank walls. At some time (again dependent upon body size) a large outward radial velocity is observed. This radial motion persists, in addition to vortical motions which decrease in size as the flow field evolves. Near the tank walls, and early in the evolution, the flow largely appears to be a collection of random events which have a general sense of motion in the direction of rotation. At essentially the same time that the fluid begins to move in a concerted $\theta$-direction near the body, the fluid near the wall exhibits the same behaviour. The radial outflow persists throughout the entire evolution process.

\section{Evolution of the tangential velocity fluctuations}

MTV measurements were conducted in order to quantify the flow visualization observations, and to correlate the behaviour of the transient torque measurements with the evolution of the velocity field. Both small and large tank data are presented. For all of the bodies investigated, time series of the tangential velocity profiles were obtained at two locations. The first measurement location resulted in profiles extending from the rotating bluff body outward into the bulk flow and the second location resulted in profiles extending from the tank wall inward. Both locations were at body and tank mid-height. Each time series of velocity profiles describes the evolution of the tangential velocity along that line from start-up until mean flow steady state is obtained. At both locations, and in both tanks, the profiles were approximately equal to $0.2 R$ in length and a typical set of profiles consisted of between 1000 and 10000 images depending on the body geometry and tank size. A short time averaging scheme was used to return evolving 'average' and fluctuating components of the tangential velocity at each pixel location from $r=L$ to $r=L+0.2 R$ near the body and from $r=R$ to $r=R-0.2 R$ near the tank wall. Averaging was done over one revolution where the total transient period ranged from 30 to 300 revolutions, dependent on $L^{*}$. Note that the flow field of interest is non-stationary. Thus, this averaging methodology does not return an average velocity as in stationary flows, nor should the fluctuating component of the velocity be termed a turbulent intensity. Ideally, statistics are computed over multiple ensembles of the start-up process. However, ensemble averaging for the flow field of interest would be impractical from a data storage perspective and the velocity fluctuations, computed as described above, do provide a representative measure of the intensity of the body-generated separated flow and turbulent motions. This is illustrated by the data of figure 2 .

Shown in figure $2(a)$ are normalized $\left(V^{*}=V /(\omega L)\right)$ tangential velocity profiles plotted vs. $r^{*}=r / R$ at different times in the transient process. The data correspond 
to $L^{*}=0.39$ in the big tank where the profiles extend from $r^{*}=0.39$ to $r^{*}=0.63$. Profiles are shown for $t^{*}=5,21,32$, and 81 where averaging was conducted over one revolution for each profile. The $t^{*}$ noted on the figure represents the temporal midpoint of the averaging scheme. For example the $t^{*}=5$ data represent an average of the data from $t^{*}=4.5$ to $t^{*}=5.5$. Also shown on figure $2(a)$ are a $V^{*}$ profile averaged over 20 revolutions after a mean flow steady state was obtained and a $V^{*}$ profile averaged over 10 revolutions during the build-up regime described by the torque measurements. The reason that averaging was done over 10 revolutions in the build-up regime is due to observations of the measured torque showing no variation in the torque during this time. Velocity measurements also show the same behaviour. Note that the $t^{*}=81$ data, although computed over 1 revolution, are only slightly different from the steady-state profile and do not exhibit large fluctuations. Similarly, the data for $t^{*}=5$ are close to the data computed over 10 revolutions in the build-up regime. The differences are greater for these data than for the steady-state data because the flow field in general is experiencing much larger velocity gradients and fluctuations at early time. The importance of these comparisons is that although the short time averaging does not return exact values of the mean velocity, it does return representative profiles of the evolving $V^{*}$ data that can be used to describe the temporal evolution of the flow field. Of interest is the fact that at steady state, $V^{*}$ at $r^{*}=L^{*}$ is slightly greater than unity (1.2), which indicates that the fluid velocity at the body corner is on average slightly greater than $\omega L$. Figure $2(b)$ plots $V^{*}$ vs. $r^{*}$ at the same $t^{*}$ as shown in figure 2(a) except that these profiles are near the tank walls. Note from figure $2(b)$ that at $r^{*}=1.0, V^{*}$ goes to zero as a consequence of the no-slip condition at the tank walls.

Normalized velocity fluctuation $\left(v^{*}=v^{\prime} /(\omega L)\right)$ profiles are also compared with $v^{*}$ profiles obtained over many revolutions at steady state and during the build-up regime in figure $2(\mathrm{c})$. These data also show that the short time averaging scheme returns values of $v^{*}$ that, while not exact, are representative of the $v^{*}$ evolution with time. At steady state and during the build-up regime, the maximum variation between the profiles shown is $10-15 \%$. These, and all of the $v^{*}$ vs. $r^{*}$ profiles, show that over the regions from $r^{*}=L^{*}$ to $r^{*}=L^{*}+0.2$ (see figure $2 c$ ) and from $r^{*}=0.75$ to 0.98 the magnitude of $v^{*}$ exhibited little variation. Thus, the $v^{*}$ values were averaged spatially over a short segment of the MTV line to provide a robust description of the evolving velocity fluctuations. This spatial averaging was done from $r^{*}=L^{*}$ to $r^{*}=L^{*}+0.1$ for the profiles near the body and from $r^{*}=0.85$ to $r^{*}=0.95$ for the profiles at the tank walls. Note that for $0.95 \leqslant r^{*} \leqslant 1.0, v^{*}$ increases significantly followed by a decreases toward zero at the tank wall. The result of this spatial averaging of $v^{*}$ was a representative value of $v^{*}$ in the region near the rotating body $\left(r^{*}=L^{*}+0.05\right)$ and near the tank wall $\left(r^{*}=0.90\right)$ for the corresponding $t^{*}$ after motion was initiated.

\subsection{Small tank evolution of $v^{*}$}

The evolution of $v^{\prime}$ at $r^{*}=L^{*}+0.05=0.57$ with time is presented in figure $3(a)$ for $L^{*}=0.52$ in the small tank. Similarly, $v^{*}$ vs. $t^{*}$ is plotted in figure $3(b)$. The acceleration, $\alpha$, was very fast $\left(10\right.$ r.p.s. $\left.{ }^{2}\right)$ for all small tank data presented. A comparison of figures $3(a)$ and $3(b)$ reinforces the flow visualization observations and torque measurements that for fixed body size the appropriate time scale is the inverse of the rotation rate. When $t$ is normalized by $1 / \omega$, the point at which $v^{*}$ starts to decay ( $t^{*} \approx 4$ revolutions) and the point at which $v^{*}$ levels off $\left(t^{*} \approx 20\right)$ are approximately the same for all $R e$. Likewise, normalization of $v^{\prime}$ by $\omega L$ merges the data along the vertical axis almost onto a single curve, with slight variation. The reason for the variation between data 

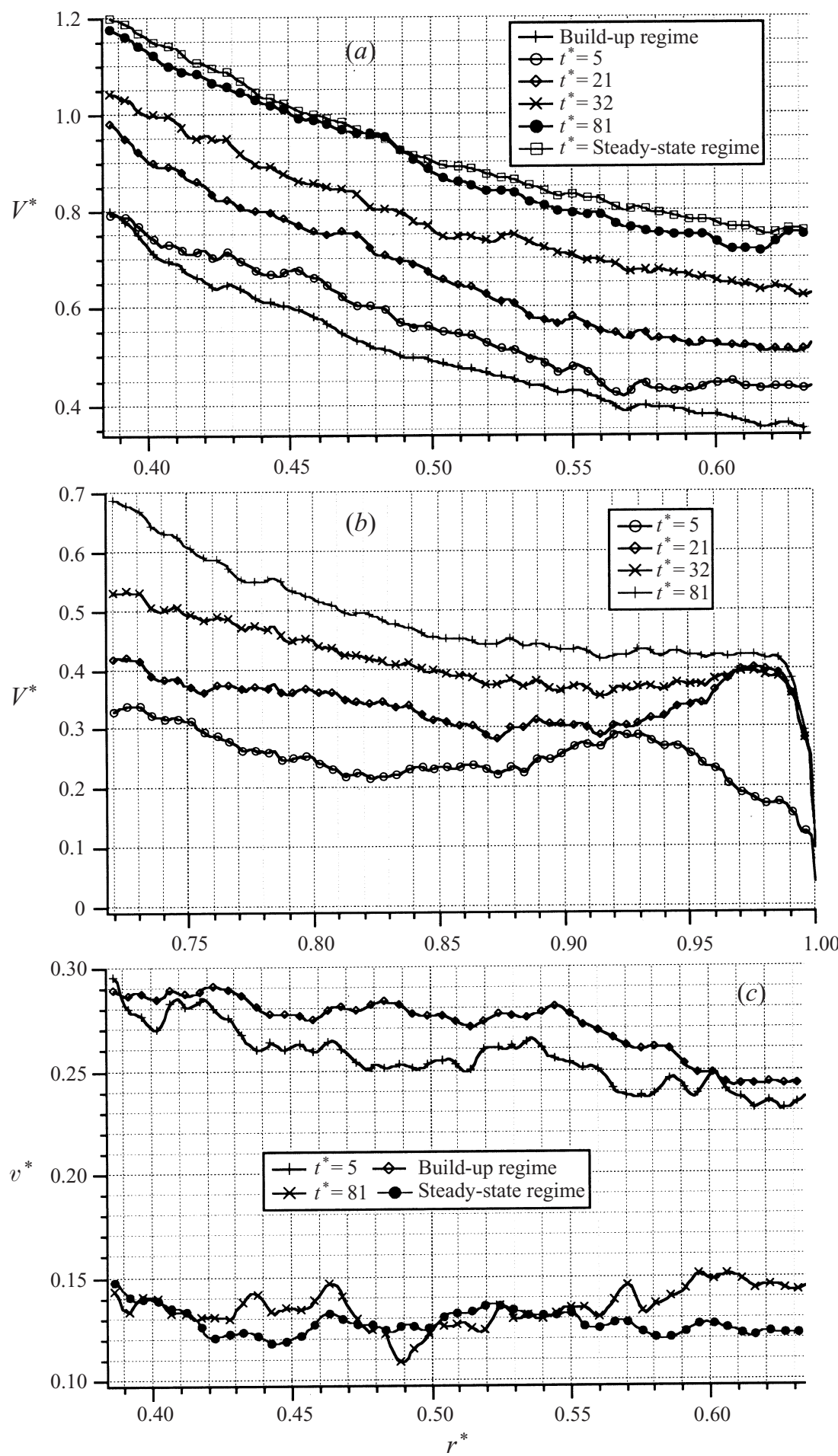

FIGURE 2. Profiles of the tangential velocity $V^{*}$, averaged over 1 revolution at four $t^{*}$ during the transient process. Shown for comparison are average profiles at steady state and during the build-up regime. Profiles are for $L^{*}=0.39$ and $R e=2 \times 10^{5}$ and extend $(a)$ from $r^{*}=L^{*}\left(r^{*}=0.39\right)$ to $r^{*}=L^{*}+0.24\left(r^{*}=0.63\right)$ and $(b)$ from $r^{*}=0.72$ to $r^{*}=1.0$. (c) Profiles of the tangential velocity fluctuations, $v^{*}$, during the build-up and steady-state regimes. 


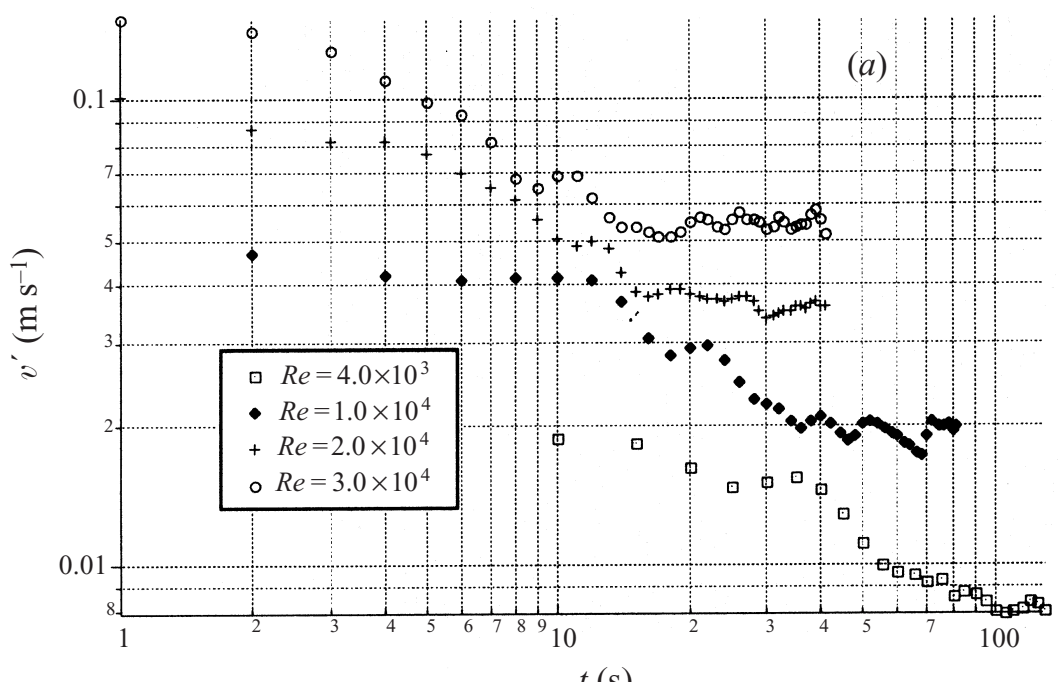

$t(\mathrm{~s})$

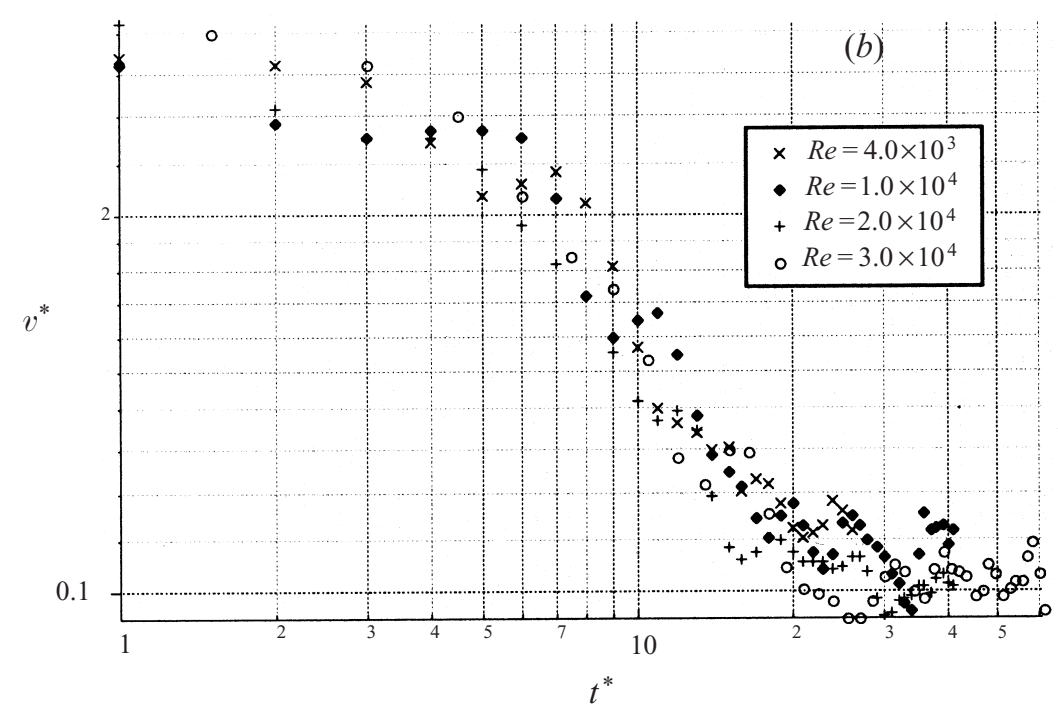

Figure 3. (a) $v^{\prime}$ vs. time at $r^{*}=0.57$ for $R e=4 \times 10^{3}, 1 \times 10^{4}, 2 \times 10^{4}$ and $3 \times 10^{4}$ with $L^{*}=0.52$ in the small tank and $(b)$ the normalized velocity flucutations, $v^{*}$, vs. $t^{*}$ for the same conditions.

sets in figure $3(b)$ cannot be determined from the present data as it may be physical or may result from the computation of $v^{*}$ as described above.

The data of figure 2 exhibit similar behaviour to the torque data (Maynes et al. 1998) wherein three distinct temporal regimes were observed. For the data of figure $3(b)$ the build-up regime corresponds to $t^{*}<4.0$. The decay regime is characterized by $v^{*}$ decaying with a power-law relation between $t^{*} \approx 4$ and $t^{*} \approx 20$. Steady state is characterized by $v^{*}$ levelling off to approximately a constant value. In the build-up regime, $v^{*}$ is about 0.28 and decreases to about 0.1 at steady state.

Figure 4 plots $v^{*}$, at $r^{*}=L^{*}+0.05$, vs. $t^{*}$ for $L^{*}=0.52$ and $L^{*}=0.65$ in the small tank. For the $L^{*}=0.65$ data, $v^{*}$ begins decaying almost immediately, indicating that the build-up regime for this $L^{*}$ is short. The value of $v^{*}$ is initially about 0.3 . This 


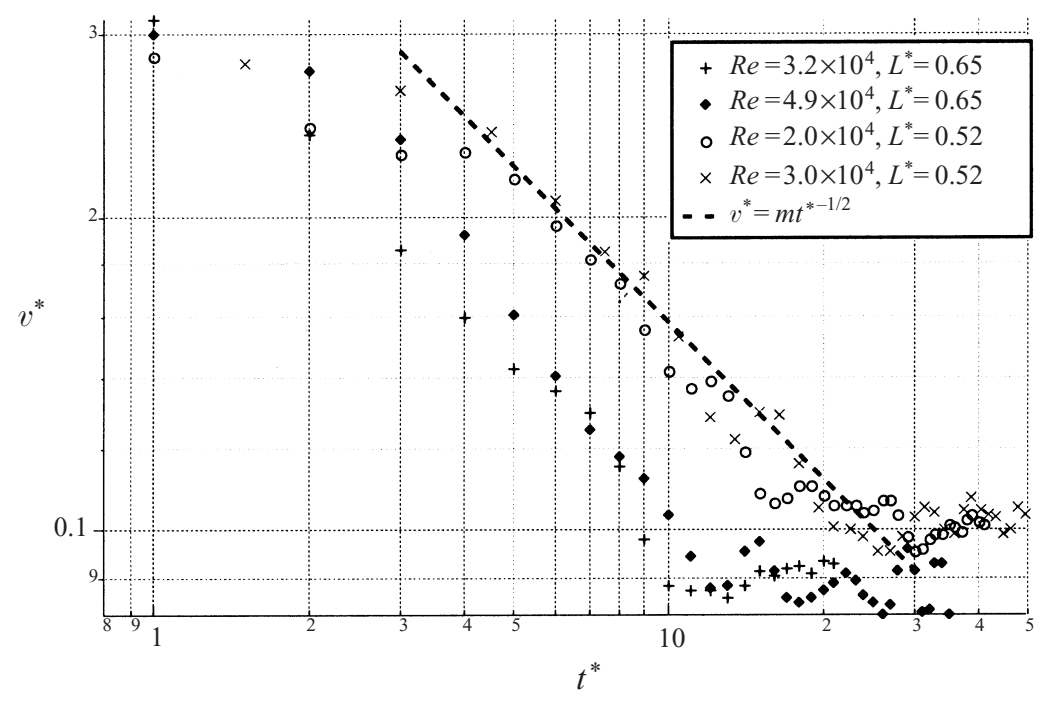

Figure 4. $v^{*}$ vs. $t^{*}$ at $r^{*}=L^{*}+0.05$ for $R e=3.2 \times 10^{4}$ and $4.9 \times 10^{4}$ with $L^{*}=0.65$, and $R e=2.0 \times 10^{4}$ and $1.5 \times 10^{4}$ with $L^{*}=0.52$ in the small tank.

is nearly the same value as $v^{*}$ for the $L^{*}=0.52$ data in the build-up regime. For this case $v^{*}$ decays to a steady-state value of about 0.09 at $t^{*} \approx 11$. The decay of $v^{*}$ proceeds at a rate similar to the $L^{*}=0.52$ data and a power-law relation is plotted on the figure indicating that the exponent during the decay regime is $\approx-1 / 2$. The data show that for $L^{*}=0.65$, steady state is attained in slightly more than half the number of revolutions as for the $L^{*}=0.52$ case. The body sizes $\left(L^{*}\right)$, however, differ only by the ratio 1.27 .

\subsection{Large tank evolution of $v^{*}$}

Data are now presented for the evolution of $v^{*}$ in the big tank as a function of $R e$, $L^{*}$ and $\alpha^{*}$. Displayed in figure $5(a)$ is $v^{*}$ vs. $t^{*}$ for $L^{*}=0.23$ with data at $r^{*}=0.28$ and $r^{*}=0.90$. For these data, the build-up regime lasts until $t^{*} \approx 50$ and steady state occurs at $t^{*} \approx 180$. Note that the value of $v^{*}$ at $r^{*}=0.28$ is about 0.23 in the build-up regime. This is smaller than the $L^{*}=0.52$ and $L^{*}=0.65$ small tank data where it was close to 0.29 . The magnitude of $v^{*}$ at $r^{*}=0.90$ is also smaller than $v^{*}$ near the body. In the build-up regime the value of $v^{*}$ near the tank wall is about 0.12 . At steady state the magnitude of $v^{*}$ near the body is about 0.13 and near the tank wall it is about 0.085 . Note, however, that the temporal behaviour of $v^{*}$ is similar near the tank wall and near the body over the range of $t^{*}$ explored.

Figure 5(b) displays $v^{*}$ vs. $t^{*}$ near the rotating body for $L^{*}=0.23$ and for $\alpha^{*}=0.04$, 0.4 and 4.0. Recall that $v^{*}$ is $v^{\prime}$ normalized by the instantaneous rotation rate, $\omega(t)$. The intensity of the velocity fluctuations, or $v^{*}$, is directly proportional to the instantaneous rotation rate. These data decay at a rate proportional to $t^{*-1 / 2}$, which is similar to the decay in the small tank and is shown by the power-law relation on the figure. Although the difference in $\alpha^{*}$ represents two orders of magnitude, the normalized data reduce to almost the same curve where transition from the build-up regime to the decay regime, and from the decay regime to steady state occur at the same $t^{*}$. If $\alpha^{*}$ is too small, however, decay will not begin at the same $t^{*}$ but will be delayed until the body has stopped accelerating. This is illustrated by the $L^{*}=0.7$ data of figure 6 where $v^{*}$ vs. $t^{*}$ is plotted for $\alpha^{*}=0.04,1.0$, and 4 at $r^{*}=0.75$. As shown by figure 

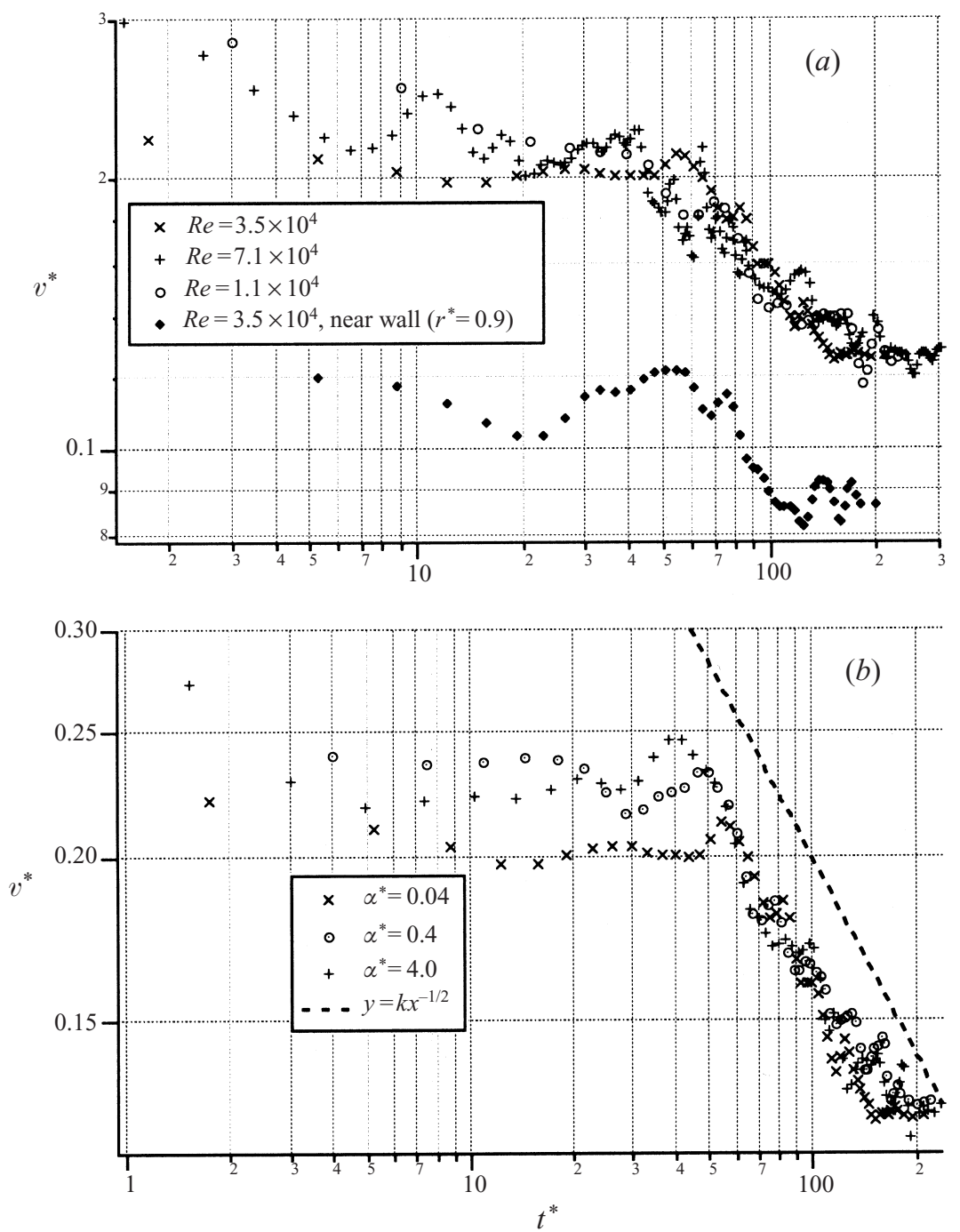

FiguRE 5. (a) $v^{*}$ vs. $t^{*}$ at $R e=3.5 \times 10^{4}, 7.1 \times 10^{4}$ and $1.1 \times 10^{4}$ at $r^{*}=0.28$ and $R e=3.5 \times 10^{4}$ at $r^{*}=0.90$ for $L^{*}=0.23$ in the big tank and $(b) v^{*}$ vs. $t^{*}$ at $R e=3.5 \times 10^{4}$ and $\alpha^{*}=0.04,0.4$ and 4.0 at $r^{*}=0.28$ for the same $L^{*}$.

6 , the case of $\alpha^{*}=0.04$ is significantly different from either of the other cases. The point at which the decay of $v^{*}$ starts is $t^{*} \approx 5$ for both $\alpha^{*}=1.0$ and $\alpha^{*}=4.0$. For $\alpha^{*}=0.04$, however, $v^{*}$ does not start to decay until $t^{*} \approx 10$. This results from the fact that the body is accelerating over 12.5 revolutions. Thus, for the case of $\alpha^{*}=0.04$, at $t^{*}=5$ revolutions, when $v^{*}$ starts to decay for the other two cases because the angular rotation rate is increasing, the magnitude of the velocity induced by the body is also increasing. Prior to the decay regime this would tend to increase the velocity fluctuations. However, because the $t^{*}$ at which decay occurs has been attained, this effect is in competition with the decrease in $v^{*}$ caused by the global features of the flow field as shown by the decay region. The result of these competing effects is a shift to larger $t^{*}$ when $v^{*}$ will begin to decay. A similar effect was observed in the torque measurements (Maynes et al. 1998). From these torque measurements it was shown 


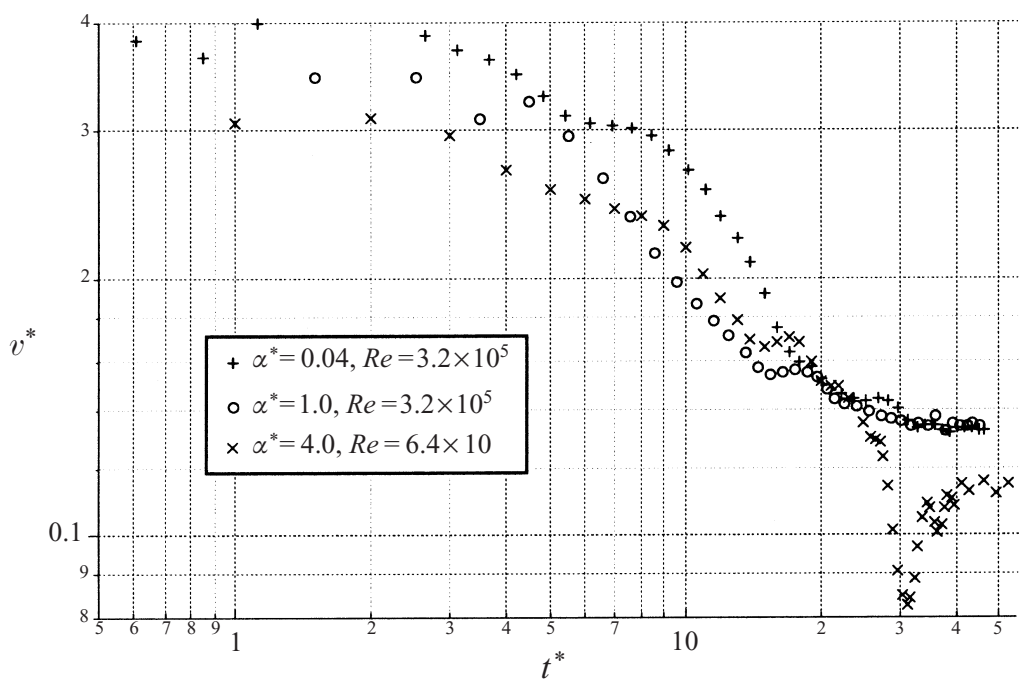

FIGURE 6. (a) $v^{*}$ vs. $t^{*}$ at $r^{*}=0.75$ with $R e=3.2 \times 10^{5}$ and $6.4 \times 10^{5}, \alpha^{*}=0.04,1.0$, and 4.0 , and $L^{*}=0.7$ in the big tank.

that if the ratio $1 / \alpha^{*}=\omega^{2} / \alpha$ (units of revolutions $(\mathrm{rev} / \mathrm{s})^{2} /\left(\mathrm{rev} / \mathrm{s}^{2}\right)$ ) is smaller than the $t^{*}$ at which decay would begin for a fast acceleration, decay begins at a fixed $t^{*}$. If $1 / \alpha^{*}$ is larger than this $t^{*}$, however, decay begins when the body stops accelerating. For the data of figure 5, decay is initiated at the same point for all $\alpha^{*}$ because, even for $\alpha^{*}=0.04$, the body has stopped accelerating prior to the $t^{*}$ at which dacay starts. This is in contrast to the $\alpha^{*}=0.04$ data of figure 6 . Note also from figure 6 that the level of $v^{*}$ in the build-up regime is about 0.3 for $\alpha^{*}=1.0$ and 4.0. This value is similar to the values of $v^{*}$ in the build-up regime for $L^{*}=0.52$ and 0.65 in the small tank. For the $\alpha^{*}=0.04$ case, $v^{*}$ in the build-up regime is closer to 0.40 . At steady state, the value of $v^{*}$ for $R e=6.4 \times 10^{5}$ is about 0.12 and is slightly greater $(\approx 0.13)$ for $\operatorname{Re}=3.2 \times 10^{5}$.

Figure 7 presents data for $L^{*}=0.39$ in the big tank. These results are similar to those for $L^{*}=0.23$ and $L^{*}=0.7$. The value of $v^{*}$ in the build-up regime is about 0.27 at $r^{*}=0.44$ and about 0.17 at $r^{*}=0.90$. The values of $v^{*}$ at steady state are about 0.12 and 0.085 for the $r^{*}=0.44$ and $r^{*}=0.90$ data respectively. It is interesting to note that at $t^{*} \approx 2$, the fluid near the tank wall has already been affected by the body motion and has reached the maximum level of $v^{*}$ observed near the tank walls. Decay starts at $t^{*} \approx 16$ and achieves steady state at $t^{*} \approx 55$ for all of the data of figure 7 . For all $L^{*}$, and in both tanks, $v^{*}$ ranges between 0.23 and 0.30 in the build-up regime and ranges between 0.09 and 0.13 at steady state. This is demonstrated in table 1 which shows typical values of $v^{*}$ in the build-up and steady-state regimes for the five $L^{*}$ discussed.

\section{Characteristic time scale development}

Also shown in table 1 are the values of $t^{*}$ where the decay and steady-state regimes begin for $L^{*}=0.23,0.39$, and 0.7 in the big tank and $L^{*}=0.52$ and 0.65 in the small tank. A characteristic time scale which collapses the $v^{*}$ data from the preceding figures onto a similar curve is sought. As mentioned previously, and shown from table 2 the 


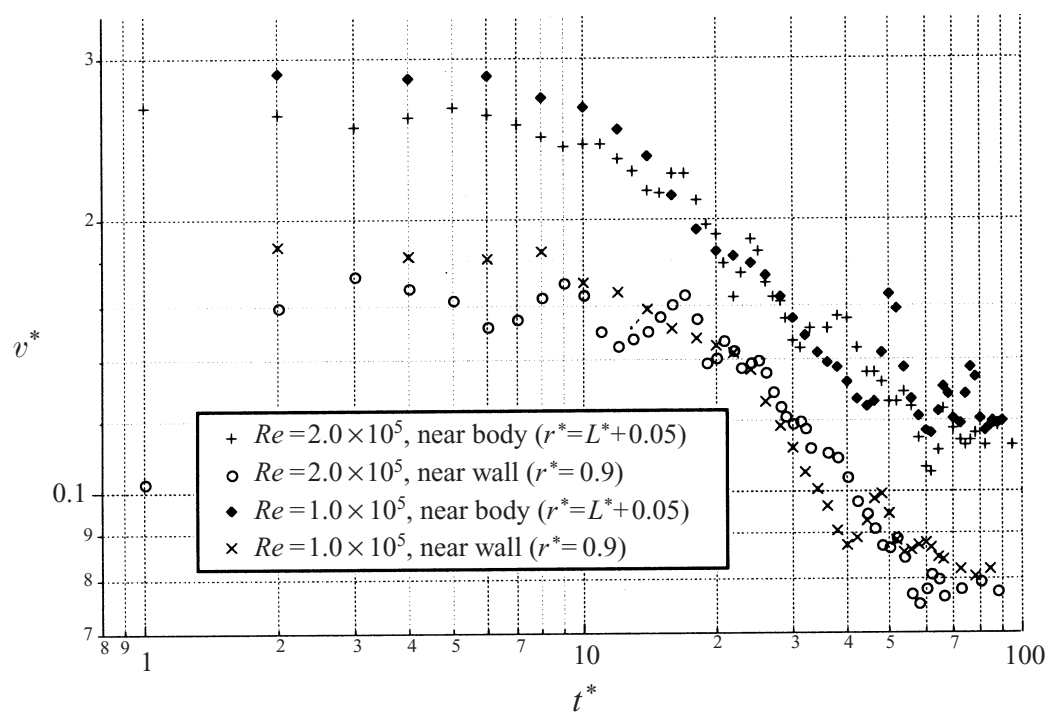

FIGURE 7. $v^{*}$ vs. $t^{*}$ at $R e=9.8 \times 10^{4}$ and $2 \times 10^{5}$ at $r^{*}=L^{*}+0.05$ and $r^{*}=0.94$ for $L^{*}=0.39$ in the big tank.

\begin{tabular}{lcccc}
\hline \multicolumn{1}{c}{$L^{*}$} & $t^{*}$ (decay) & $t^{*}$ (steady state) & $v^{*}$ (decay) & $v^{*}$ (steady state) \\
0.52 (small tank) & 4 & 18 & 0.28 & 0.1 \\
0.65 (small tank) & 2 & 10 & 0.30 & 0.09 \\
0.23 (big tank) & 45 & 200 & 0.23 & 0.13 \\
0.39 (big tank) & 16 & 75 & 0.27 & 0.12 \\
0.7 (big tank) & 5 & 26 & 0.28 & $0.12-0.13$
\end{tabular}

TABLE 1 . Values of $v^{*}$ and $t^{*}$ marking the beginning of decay and steady-state regimes.

$t^{*}$ when the decay and steady-state regimes occur vary with body geometry. This fact holds even for similar $L^{*}$ but different $H^{*}(H / R)$. For example, with $L^{*}=0.7$ in the big tank, the $t^{*}$ at which steady state is attained occurs at about 26. By contrast for $L^{*}=0.65$ in the small tank, the $t^{*}$ for steady state is about 11 . Neglecting Re and $\alpha^{*}$ effects, $v^{*}$ is a function only of $t^{*}$ and the geometric parameters $L^{*}, h^{*}$, and $H^{*}$. By simply combining these terms, with some trial and error, a non-dimensional time scale can be developed which causes the data to merge within a factor of 2 . Representative data from the five $L^{*}$ previously discussed are plotted on figure $8(a)$ using this time normalization. For all of the data plotted on figure 8(a) the accelerations were fast. Physically, this time scale represents the number of revolutions multiplied by the ratio of bluff body volume to tank volume and is written as $t_{d}=t^{*}\left(L^{2} / R\right)(h / H)$. Use of this time scaling successfully causes all of the big tank data to begin to decay at a fixed $t_{d}$ and to attain steady state at a different but fixed value of $t_{d}$ : the small tank data behave similarly. The $t_{d}$ when steady state is attained, however, is different for the two tanks: the values differ by about a factor of 2 . If the small and big tanks were geometrically similar these values would probably be the same. This time scaling was used to collapse the torque data from the large tank such that the decay and steady-state regimes occur at fixed values of $t_{d}$ regardless of the body size. A time 

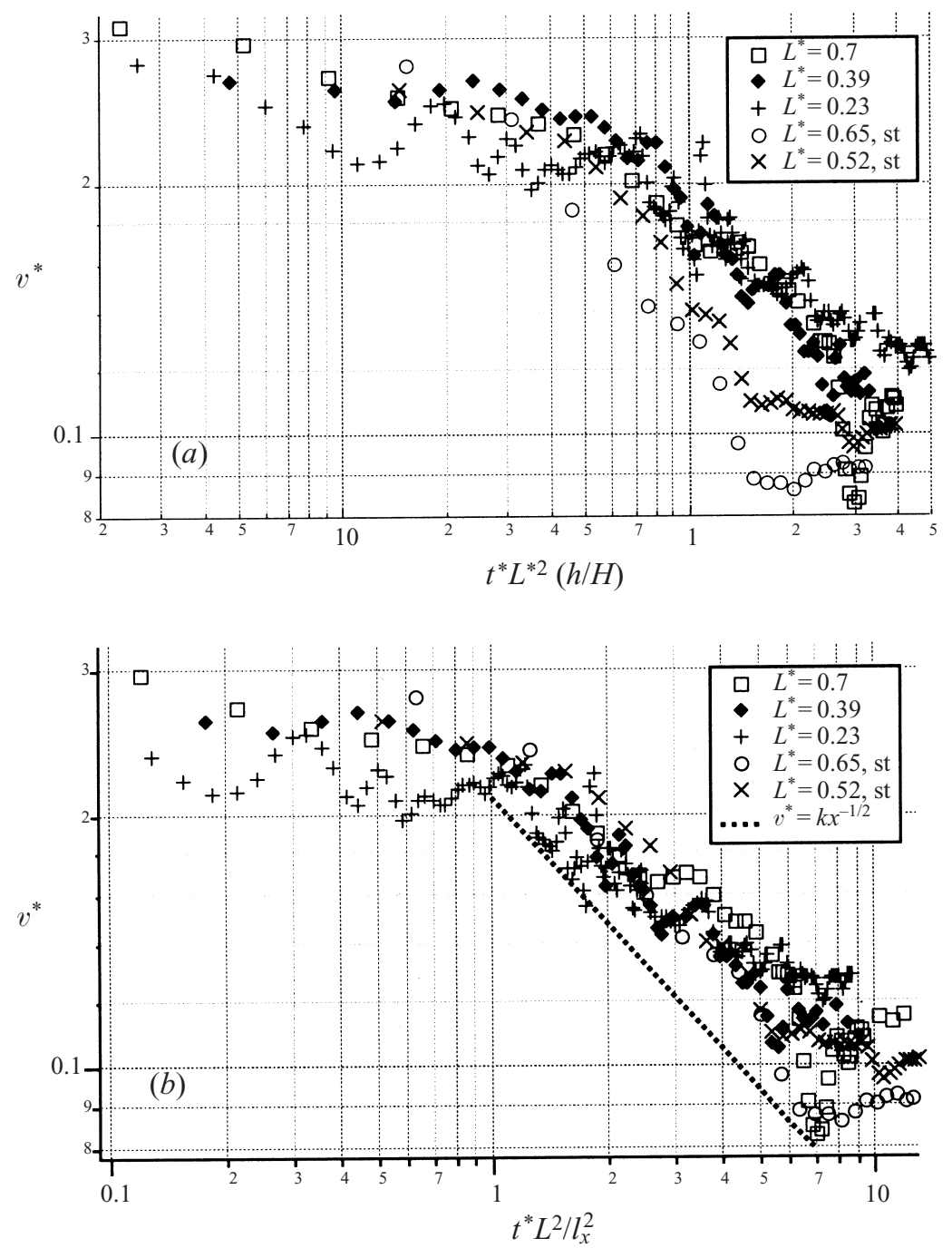

FiguRE 8. (a) $v^{*}$ vs. $t_{d}$ for $L^{*}=0.52, R e=2 \times 10^{4}$ and $L^{*}=0.65, R e=3.1 \times 10^{4}$ in the small tank and $L^{*}=0.23,0.39$, and 0.70 in the big tank. The respective $R e$ values in the big tank are $R e=7.1 \times 10^{4}, 2 \times 10^{5}$, and $6.4 \times 10^{5}$. (b) $v^{*}$ vs. $\tau$ for the same cases as in $(a)$.

scale based upon the characteristic time required for turbulent motions to convect a given distance is now developed to merge the data from the two tanks.

Standard scaling for turbulent flow (Tennekes \& Lumley 1994) indicates that the time for mixing over some distance $l_{x}$ is $t_{x}=l_{x}^{2} / D_{t}$, where $D_{t}$ is a turbulent diffusivity. $D_{t}$ scales like $U l$ where $U$ and $l$ are characteristic velocity and length scales of the turbulence. In the case of a rotating bluff body the length scale of the dominant turbulent motions, $l$, is of the order of the length of a body side, and the characteristic velocity $U$ should scale with the velocity of a body corner which is $\omega L$. Substituting $\omega L^{2}$ for $D_{t}$ into the expression for $t_{x}$ above and normalizing time by $t_{x}$ results in the non-dimensional time scale $\tau=\omega t L^{2} / l_{x}^{2}$. For the case of high $\alpha$, $\omega t$ is simply revolutions; thus $\tau=t^{*} L^{2} / l_{x}^{2}$. This scaling contains revolutions, characteristic body size, $L$, and tank, $l_{x}$, length scale information. For the case of two infinitely long 


\begin{tabular}{ccccccc}
\hline (a) $\quad \omega($ r.p.s.) & Time $(\mathrm{s})$ & $\tau$ & $(b)$ & $\omega$ (r.p.s.) & Time $(\mathrm{s})$ & $\tau$ \\
0.3 & 36.75 & 0.985 & 0.5 & 65.2 & 0.931 \\
0.3 & 35.09 & 0.9408 & 0.5 & 66.3 & 0.946 \\
0.4 & 23.38 & 0.836 & 1.0 & 29.3 & 0.836 \\
0.4 & 25.6 & 0.915 & 1.0 & 29.8 & 0.850 \\
0.5 & 20.73 & 0.926 & 1.0 & 31.3 & 0.894 \\
0.5 & 21.16 & 0.945 & 1.5 & 20.0 & 0.827 \\
0.6 & 17.88 & 0.958 & 2 & 16.9 & 0.967 \\
0.6 & 16.38 & 0.878 & & & 16.0 & 0.911 \\
0.7 & 12.93 & 0.81 & & & \\
0.7 & 12.37 & 0.774 & & & \\
0.8 & 11.94 & 0.854 & & & & \\
0.8 & 11.38 & 0.814 & & & &
\end{tabular}

TABLE 2. Time at which surface float starts moving: $(a) L^{*}=0.39$ (average $\tau=0.890$ )

(b) $L^{*}=0.23$ (average $\tau=0.891$ ).

concentric cylinders the obvious choice for $l_{x}$ is the distance between cylinders $R-L$. Mixing tanks in general are of finite length; therefore a characteristic length scale based on both the radius and height of the tank is required. The longest dimension in the tank, as measured from the body surface to the outer tank wall, represents the greatest distance that turbulence diffuses or convects. At the time when turbulence has diffused over this distance, the entire flow field has been affected by the motion of the rotating body. This distance is a function of the tank height and radius and body height and face length and is taken to be the distance from the body to the upper corner of the flow field (see figure 1); $l_{x}^{2}$ is expressed as the square of this distance as

$$
l_{x}^{2}=\left(\frac{1}{2} H-\frac{1}{2} h\right)^{2}+(R-L)^{2} .
$$

Thus, $t_{x}$ represents the time scale required for the turbulent diffusion process to act over a characteristic length and is completely analogous to the time scale based on molecular diffusion in a laminar flow. By selecting this length as the longest dimension in the tank over which turbulence can propagate, $t_{x}$ represents the time required for turbulent motions to fill the tank. Substituting (1) into the expression for $\tau$ results in an expression for the non-dimensional time as

$$
\tau=t w(t) \frac{L^{2}}{\left(\frac{1}{2} H-\frac{1}{2} h\right)^{2}+(R-L)^{2}} .
$$

If the acceleration is fast, the term $\omega t$ represents the number of revolutions of the body. In general, $\omega$ is a function of time, $\omega(t)$, and because $\tau$ depends on the turbulent motions generated as $\omega$ increases or decreases, (2) should be integrated over time to account for the cumulative 'history' effects. Recognizing that $\omega=\alpha t$, integration over time of this term also yields the number of revolutions of the body. Thus, the term $\omega t$ can be replaced by $t^{*}$ for all $\omega(t)$ and (2) can be rewritten as a function of the non-dimensional parameters:

$$
\tau=t^{*} \frac{L^{* 2}}{\left(\frac{1}{2} H^{*}-\frac{1}{2} h^{*}\right)^{2}+\left(1-L^{*}\right)^{2}} .
$$

The expression for $\tau$ in (3) is general for any tank size and body size. If the body has 
the same height as the tank, $\tau$ is a function of $t^{*}$ and $L^{*}$. Likewise if the body has a small height but has a radius close to $R, \tau$ would be a function only of $t^{*}, H^{*}$ and $h^{*}$.

The $v^{*}$ data of figure $8(a)$ are plotted vs. $\tau$ in figure $8(b)$. It is apparent from figure $8(b)$ that $\tau$ is the appropriate time scaling for this flow as both the small tank and big tank data fall on almost the same curve. Further evidence that this is the appropriate time scale lies in the fact that the decay region starts at about $\tau=1$. Recall that $\tau$ represents the ratio of actual time to the time required for turbulent motions to convect over the tank volume. Thus, it is expected that at about $\tau=1$ turbulent motions, and thus some angular momentum, will have been convected throughout tank, leading to a decrease in the velocity fluctuations. By replacing $\tau$ with 1.0 in (3) we can solve for the number of revolutions required, for a given tank and body, to fill the tank with turbulent motions and when decay begins.

Of particular significance regarding the non-dimensional time scaling, $\tau$, is that it merges data from two tanks differing in volume by a factor of 80 and body sizes differing in length, $L$, by a factor of 5.6. It is further believed that $\tau$ represents the appropriate non-dimensional start-up time scale for any rotating geometry inducing turbulent flow in a confined tank.

The near-wall data $\left(r^{*}=0.90\right)$ behave similarly, in a temporal sense, to the data of figure $8(b)$ when plotted vs. $\tau$. The magnitude of $v^{*}$ is smaller, however, throughout the transient process and shows much more dependence upon the body size than the $v^{*}$ data at $r^{*}=L^{*}+0.05$. This dependence is now briefly discussed. Because the characteristic turbulent motions are generated at the body owing to shear layers associated with the separated regions and shed vortices, the magnitude of $v^{*}$ is smaller near the tank wall due to the diffusion and dissipation of the turbulent motions as they convect outward. Also, the characteristic length scale of the dominant motions is proportional to $L^{*}$. Thus, as $L^{*}$ increases not only does the amount of fluid volume decrease, but the body-generated characteristic motions also represent a larger percentage of the fluid volume. Consequently, as $L^{*}$ increases, the magnitude of $v^{*}$ near the tank walls also increases as the data show.

\section{1. $C_{m}$ data evidence of a turbulent diffusion time scale}

Shown in figures $9(a)$ and $9(b)$ are data from measurements of the evolving hydrodynamic torque. Figure $9(a)$ plots the transient torque coefficient $C_{m}=T /\left(\frac{1}{2} \rho \omega^{2} L^{4} h\right)$ vs. $\tau$ for the $R e$ and $L^{*}$ listed in the figure caption. $T$ is the measured torque, $\rho$ is the fluid density, and $\omega$ is expressed in radians per second. The $C_{m}$ data presented here were obtained at an acquisition rate of $200 \mathrm{~Hz}$. The temporal behaviour is very similar to the $v^{*}$ data. $C_{m}$ is approximately constant in the build-up regime until $\tau \approx 1$. It decays between $\tau \approx 1$ and $\tau \approx 6$ where it levels off to different steady-state values. For all cases $C_{m}$ decays proportional to $\tau^{-n}$. The value of $n$ is close to 1 for $L^{*}=0.7,0.39$, and 0.23 ; but it appears to decrease slightly for the case of $L^{*}=0.31$ where $h^{*}=0.48$. Figure $9(b)$ plots $C_{m}$ vs. $\tau$ for the $L^{*}=0.15$ case and two cases with $L^{*}=0.13$. For the $L^{*}=0.13$ case denoted $\mathrm{B}$ on the figure, the body was centred in the tank and the tank was completely full. For the case denoted $\mathrm{C}$ the body was positioned $2 \mathrm{~mm}$ above the tank bottom and the water level was lowered to the top of the body and thus, the body was as tall as the fluid height. A free surface exists for this case. The rotation rates were small enough, however, that the Froude number was small and therefore free surface effects were not significant.

Figure $9(b)$ illustrates the generality of (3) where the body height and tank height for case $\mathrm{C}$ do not enter into the determination of $\tau$. The non-dimensional time when decay starts, however, is very near the value for the other cases. The magnitude of $C_{m}$ 

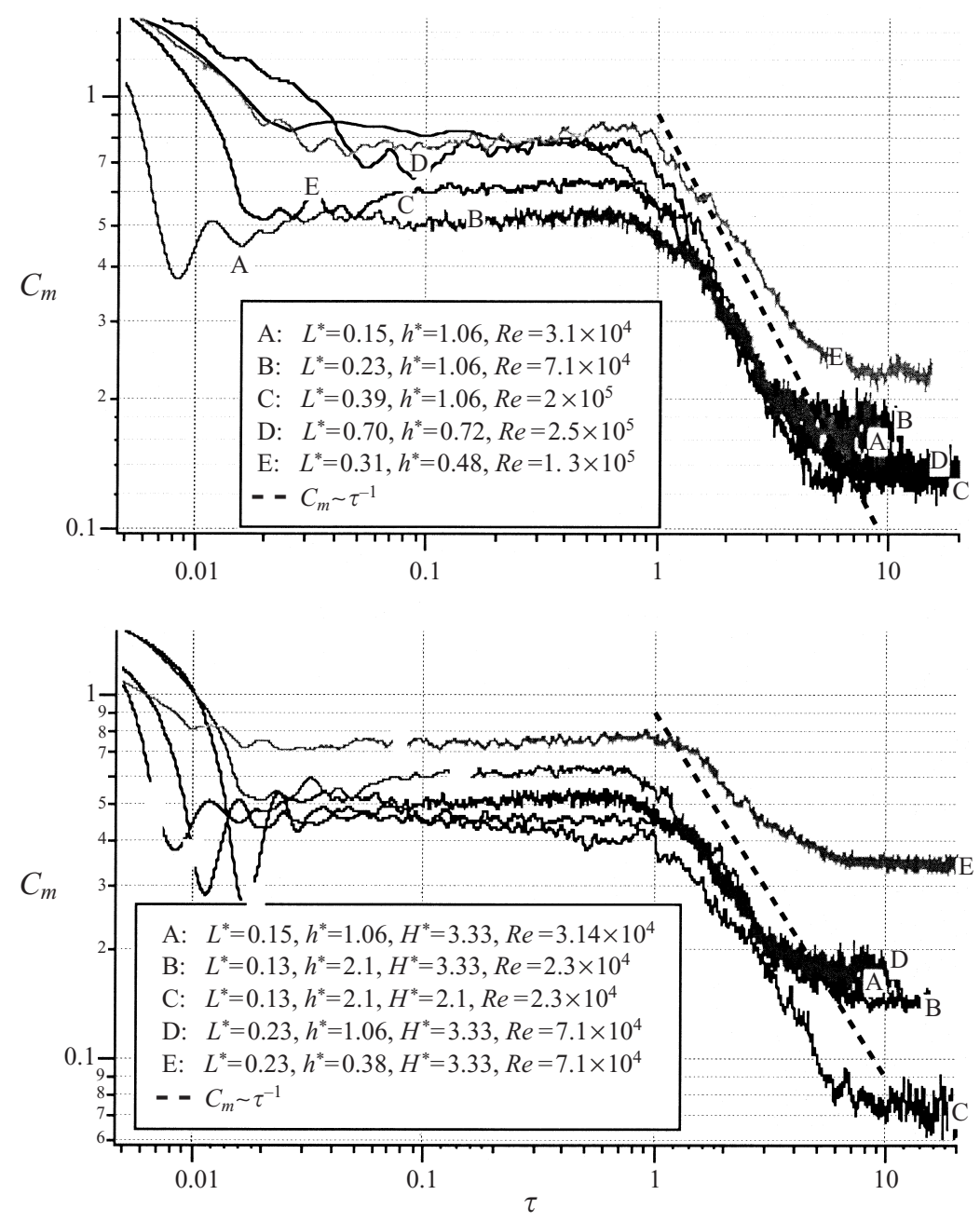

FIGURE 9. $C_{m}$ vs. $\tau$ for $(a) L^{*}=0.15$ and $h^{*}=1.06, L^{*}=0.23$ and $h^{*}=1.06, L^{*}=0.31$ and $h^{*}=0.48, L^{*}=0.39$ and $h^{*}=1.06$, and $L^{*}=0.7$ and $h^{*}=0.72$ in the big tank with $H^{*}=3.33$ and (b) $L^{*}=0.15$ and $h^{*}=1.06$, two cases with $L^{*}=0.13$ and $h^{*}=2.1$ but different $H^{*}$, and two cases with $L^{*}=0.23$ and $H^{*}=3.33$ with different $h^{*}$.

in the build-up regime is the same for both $H^{*}=3.33$ and $H^{*}=2.1$ and is slightly smaller in magnitude than the $L^{*}=0.15$ case. This indicates that the magnitude of $C_{m}$ during build-up is a function only of the rotational parameters and geometry of the body. During the build-up regime the measured torque is the same as if the body were rotating in an infinite fluid. At steady state, however, $C_{m}$ is much smaller (0.08) for the case $H^{*}=h^{*}=2.1(0.15)$, where the body extends over the height of the fluid, than for the $H^{*}=3.33$ case. The reason for this is that once decay begins the tank geometry is important and in addition to the body geometry and rotational parameters determines what steady-state condition will be attained. At steady state there exists a balance between the torque exerted on the body and the torque on the tank walls. For $H^{*}=2.1$ the steady state attained is different than for the case when $H^{*}=3.33$. This leads to a smaller $C_{m}$ for the $H^{*}=2.1$ case because the fluid height and thus viscous forces on the tank wall are lower than for the $H^{*}=3.33$ case. 
Also shown in figure $9(b)$ are two cases with $L^{*}=0.23$, one with $h^{*}=1.06$ and one with $h^{*}=0.38$. The difference in height is about 3 times; but the difference in $C_{m}$ is about a factor of 1.25 during the build-up regime and about a factor of 2.0 at steady state. An interesting trend from figures $9(a)$ and $9(b)$ is that as the ratio $L^{*} / h^{*}$ decreases, the magnitude of $C_{m}$ in both the build-up and steady-state regimes also decreases. Also as $h^{*}$ gets smaller (e.g. $L^{*}=0.31, h^{*}=0.48$ and $L^{*}=0.23, h^{*}=0.38$ ) the decay exponent, $n$, also decreases. These trends in the $C_{m}$ data deserve further investigation.

\subsection{Visualization evidence of a turbulent diffusion time scale}

A set of visualization experiments were also conducted that provide additional evidence that the turbulent diffusion time scale is the appropriate characteristic time scale of the flow. To conduct these experiments the surface of the water level was lowered to $5 \mathrm{~cm}$ below the top of the tank. A small plastic float was placed on the surface and observed as the bluff body was started into motion. The time at which the float started moving was recorded. It was also observed that just prior to motion of the float, the effects of turbulent motions were observed on the surface. These vortices, or motions, caused the surface to rapidly transition from smooth and stationary to moving and turbulent. This experiment was conducted with $L^{*}=0.23$ and 0.39 . Table 2 shows $L^{*}, \omega, t$, and $\tau$ for a portion of the data collected.

The data of table 2 further reinforce that the non-dimensional time scale, $\tau$, is the appropriate time scale of the transient flow field. For both $L^{*}=0.23$ and 0.39 the value of $\tau$ when the surface float started moving, averaged over the independent realizations, is 0.89 . This value is $11 \%$ smaller than the value from the MTV data where it appears that $v^{*}$ is beginning to decay. This is probably due to $v^{*}$ changing by an unnoticeable amount when the decay first begins.

\section{General description of the evolving flow field}

A general description of the flow field for the transient process is now given. Observations from velocity measurements and flow visualization studies by Maynes (1997) indicate that when a body begins to rotate in a quiescent fluid, large axial velocities are induced by the body owing to low-pressure regions which exist in the core of the separated flow near the body faces. In the vicinity of the body midplane, the body-induced axial velocity is converted to a radial outflow (impeller stream). The pumping of fluid axially is driven by the body and should not be confused with transport caused by the formation of an Ekman layer in a spinning tank discussed in detail by Wedemeyer (1964). This induction of axial flow in the region of the separated regions is an interesting phenomenon and should be investigated further. Initially then (during the build-up regime), the fluid which is transported in the axial direction due to the axial velocities largely consists of fluid with a component of $\theta$-momentum that is close to zero. Additionally, the flow appears to be a collection of randomly interacting eddies moving outward from the body. Because of this axial transport, the velocity gradients near the surface do not decrease, but remain large; and the regions of separated flow 4extend out to the bluff body corners as observed from flow visualization. Consequently, the tangential velocity fluctuations and hydrodynamic torque remain constant. The flow field in the vicinity of the body during the build-up regime is similar to the flow field that would occur if the body were to be rotating in an infinite fluid. The time over which this behaviour occurs is referred to as the build-up regime. 
After turbulent motions have convected over the entire tank volume, corresponding to the characteristic non-dimensional time, $\tau \sim 1$, all fluid transported by the bodydriven secondary flow has associated with it some angular momentum. As a result, the relative velocity between the rotating body and this fluid is smaller than in the build-up regime when the fluid had zero $\theta$-momentum. Subsequently, the velocity gradients and size of the separated regions near the body decrease. As a result, the magnitude of the torque and velocity fluctuations decays, exhibiting power-law relations with time where the decay exponents are close to -1 and $-1 / 2$ respectively. Because of this behaviour, this regime is referred to as the decay regime. During this same regime, the magnitude of the average tangential velocity increases because of a transfer of momentum from the body as it rotates in the fluid. Eventually steady state is attained and the mean tangential velocity at $r^{*}=L^{*}$ levels off at a constant value slightly greater than $\omega L$ and the torque coefficient and velocity fluctuations also level off.

\section{Conclusions}

The general evolution of the turbulent flow field induced by a rotating bluff body in a tank has been described from measurements of the evolving tangential velocity in conjunction with flow visualization observations and hydrodynamic torque measurements. For a bluff body starting from rest in a quiescent fluid, three temporal regimes have been observed and discussed.

Based on the concept of momentum transport due to turbulent diffusion, a time scaling has also been presented and discussed which successfully collapses data for two tank sizes differing in volume by a factor of 80 and with bluff bodies differing in characteristic length by a factor of 6 . This time scaling is general for all body sizes, tank sizes, rotation rates and acceleration rates explored, and it describes start-up in a tank for a bluff-body-driven flow. One significance of this time scale is that it suggests appropriate scale-up relations from a small tank to a large tank while maintaining fixed mixing dynamics for systems employing time-dependent rotational parameters.

\section{REFERENCES}

Bourne, J. R. \& Dell'Ava, P. 1987 Micro- and macro-mixing in stirred tank reactors of different sizes. Chem. Engng Res. Design 65, 180-186.

Costes, J. \& Couderc, J. P. 1988 Study by laser Doppler anemometry of the turbulent flow Induced by a Rushton turbine in a stirred tank: influence of the size of the units. Chem. Engng Sci. 43, $2765-2772$.

Falco, R. E. \& Chu, C. C. 1987 Measurement of two-dimensional fluid dynamic quantities using a photochromic grid tracing technique. SPIE 814, 706-710.

Gendrich, C. P. \& Koochesfahani, M. M. 1996 A spatial correlation technique of estimating velocity fields using molecular tagging velocimetry (MTV). Exps. Fluids 22, 67-77.

Greenspan, H. P. 1968 The Theory of Rotating Fluids. Cambridge University Printing House.

Gunkel, A. A. \& Weber, M. E. 1975 Flow phenomena in stirred tanks. AIChE J. 21, 931-949.

Hill, R. B. \& KLEWICKI, J. C. 1996 Data reduction methods for flow tagging velocity measurements. Exps. Fluids 20, 142-152.

MAYNES, D. 1997 On rotating bluff body flows. PhD Dissertation, University of Utah.

Maynes, D., Klewicki, J. C. \& MCMurtry, P. A. 1998 Time resolved torque of three dimensional rotating bluff bodies in a cylindrical tank. Trans. ASME: J. Fluids Engng 120, 23-29.

MiLLER, S. 1962 Photochemical reaction for the study of velocity patterns and profiles. BASc Thesis, University of Toronto. 
Ponce, A., Wong, P. A., Way, J. J. \& Nocera, D. G. 1993 Intense phosphorescence triggered by alcohols upon formation of a cyclodextrin ternary complex. J. Phys Chem. 97, 11137-11142.

Rice, R. W. \& BAUD, R. E. 1990 The role of micromixing in the scale-up of geometrically similar batch reactors. AIChE J. 36, 293-298.

Roberts, R. M., Gray, M. R., Thompson, R. \& Kresta, S. M. 1995 The effect of impeller and tank geometry on circulation time distributions in stirred tanks. Trans. Inst. Chem. Engrs A 73, 78-86.

Rutherford, K., Mahmoudi, M. S., Lee, K. C. \& Yianneskis, M. 1996. The influence of Rushton impeller blade and disk thickness on the mixing characteristics of stirred vessels. Trans. Inst. Chem. Engrs A 74, 369-378.

Tennekes, H. \& Lumley, J. L. 1994 A First Course in Turbulence. MIT Press.

WedEmeYer, E. H. 1964 The unsteady flow within a spinning cylinder. J. Fluid Mech. 20, 383-399.

Wu, H. \& PATterson, G. K. 1989 Laser-Doppler measurements of turbulent-flow parameters in a stirred tank. Chem. Engng Sci. 44, 2207-2221.

Zaitseva, N. P. 1997 Rapid growth of large-scale $(40-55 \mathrm{~cm}) \mathrm{KH}_{2} \mathrm{PO}_{4}$ crystals. J. Crys. Growth 180, 225-262.

Zhou, G. \& Kresta, S. M. 1996 Distribution of energy between convective and turbulent flow for three frequently used impellers. Trans. Inst. Chem. Engrs A 74, 379-389. 